\title{
Second Redo for Composite Graft Pseudoaneurysm with Transcutaneous Rupture
}

\author{
Mikael Péterffy, MD, Christian Olsson, MD, PhD* \\ Department of Molecular Medicine and Surgery, Cardiovascular Surgery Unit, The Karolinska Institute and Karolinska University \\ Hospital, Stockholm, Sweden
}

\begin{abstract}
Six months after a composite graft redo operation repairing two pseudoaneurysms at the distal suture line and the right coronary artery, respectively, a patient returned asymptomatic and in good general condition but with new presternal bulges. Computed tomography and angiography diagnosed a new pseudoaneurysm of the left coronary artery, and on frank rupture, acute re-repair was undertaken with the aid of presternotomy hypothermic circulatory arrest. Temporary postoperative neurological dysfunction subsided and recovery was otherwise uneventful.

Copyright @ 2014 Science International Corp.
\end{abstract}

\section{Key Words}

Aorta - Aortic pseudoaneurysm - Suture - Resternotomy

\section{Introduction}

Late-occurring pseudoaneurysms are preferably detected and evaluated by routine regular radiological follow-up and dealt with in a timely manner. Acute presentation portends a worse outcome and may mandate alternative surgical strategies.

\section{Case Presentation}

The patient, a 58-year-old man, was operated twice for aortic coarctation in childhood; he had no signs of significant re-coarctation. In 1981, he underwent composite graft aortic root replacement (Bjork-Shiley 31

\author{
(C) 2014 Aorta \\ Published by Science International Corp. \\ ISSN 2325-4637 \\ Accessible online at: \\ http://aorta.scienceinternational.org
}

$\mathrm{mm}$ ) for aortic valve endocarditis, and 31 years later, in 2012, he was reoperated, again due to suspected, culture-negative endocarditis. At reoperation, the aortic valve itself was deemed intact, fully functional, and uninfected, but two separate pseudoaneurysms due to anastomotic insufficiency were corrected at the distal anastomosis and at the reimplantation of the right coronary artery, respectively. After a course of antibiotics, the patient recovered well. Six months later, he returned in good general condition, but with two new presternal bulgings, as documented by a resident with his mobile phone camera (Fig. 1). At palpation, they were fluctuant, nontender, and very subtly discolored. Acute computed tomography (CT) scan (Fig. 2) and angiography (Fig. 3) confirmed leakage at the reimplanted left coronary artery. Surgery was scheduled for the next morning, but during the night, one of the bulges ruptured with ensuing hemorrhage, and the patient was taken immediately to the operating room.

With the patient in stable condition, the groin was cannulated and extracorporeal circulation instituted. The patient was cooled to deep $\left(18^{\circ} \mathrm{C}\right)$ hypothermia and then resternotomy was performed, with entrance into a partially thrombosed retrosternal pseudoaneurysm. The ascending graft was identified and clamped. Perfusion was restarted and cold blood cardioplegia was administered antegradely, helping to identify the bleeding at the anticipated location, cranially-dorsally in the left coronary artery anastomosis.

*Corresponding author:

Dr. Christian Olsson, MD, PhD

Thoraxkliniken

Karolinska Universitetssjukhuset

SE-17176, Stockholm, Sweden

Tel: +4685170000, Fax: +468322701, E-Mail: christian.olsson@ki.se 


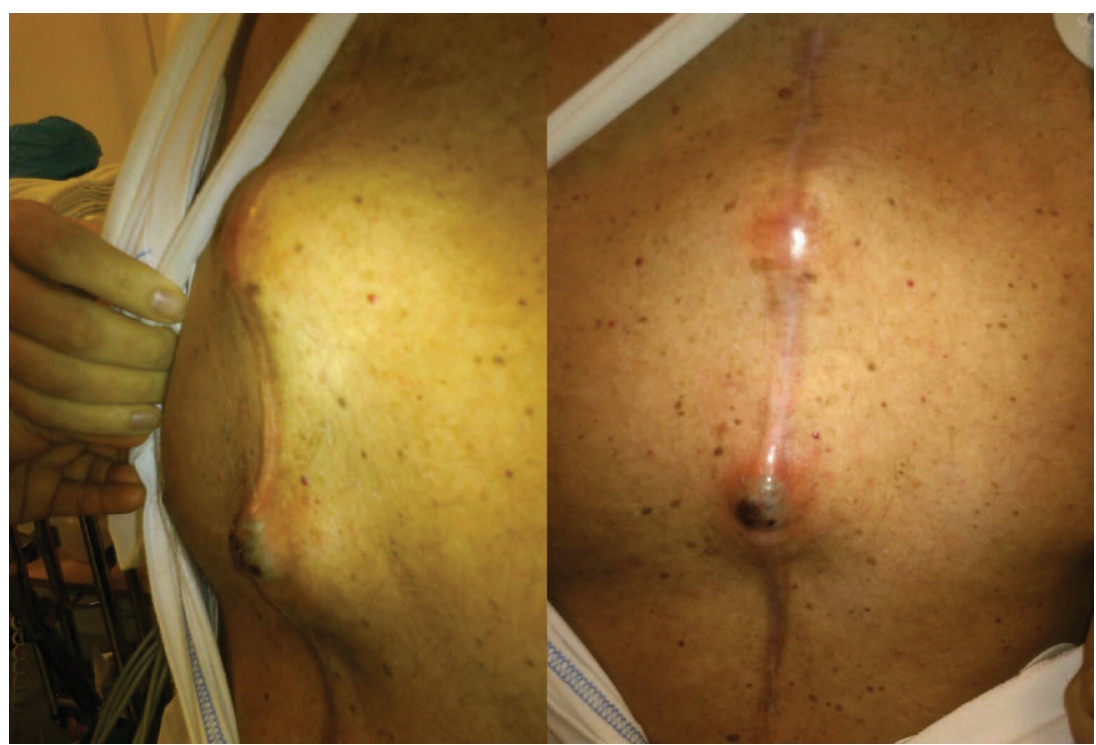

Figure 1. Mobile phone photos of the external appearance, showing two distinct bulgings in an otherwise healed sternotomy incision. Note very thin coverage over the caudal swelling, and the absence of generalized wound discoloration.

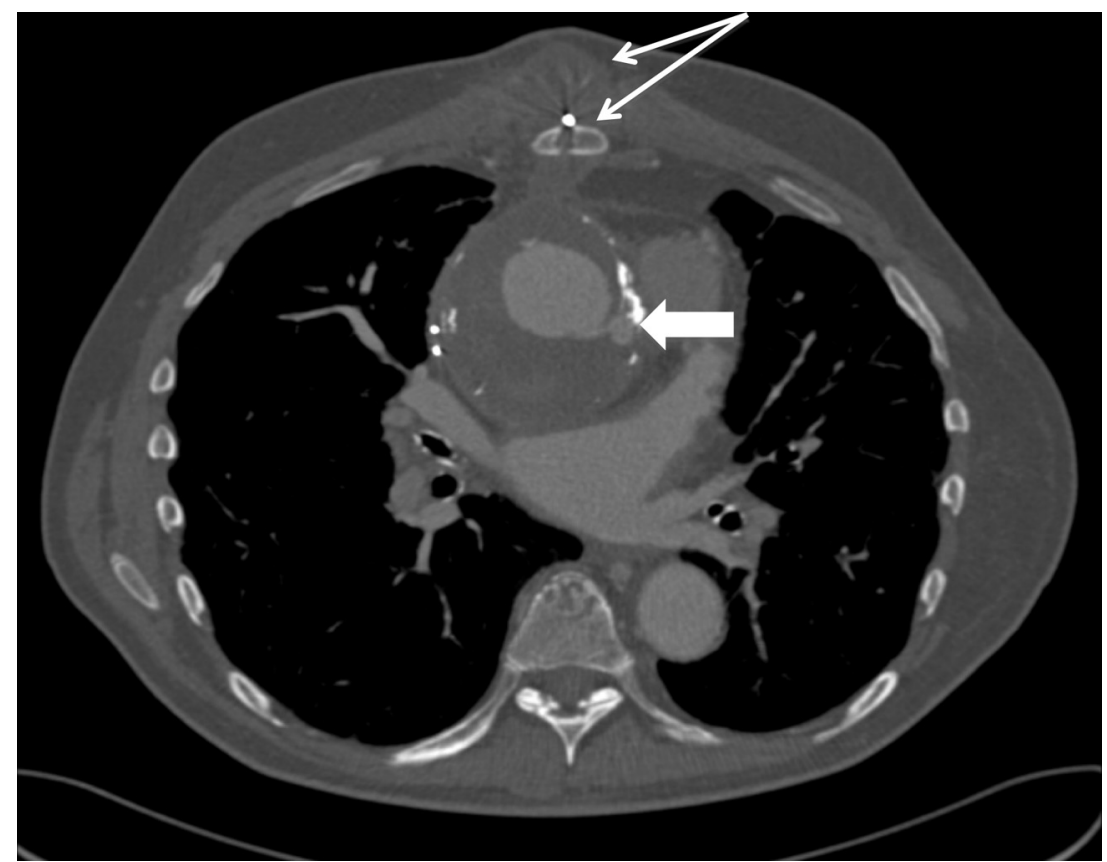

Figure 2. Computed tomography demonstrating contrast extravasation (thick white arrow; circular collection of dye next to linear calcification) close to the graft and surrounding hematoma. Thin white arrows demonstrate subcutaneous hematoma and incomplete sternal healing.

Due to the very severely calcified adhesions surrounding the aortic root and graft (Fig. 2), a decision was made to suture the defect using pledgeted $4 / 0$ monofilament suture rather than attempt a complete reanastomosis of the left coronary artery. Bleeding was controlled, the patient was rewarmed and separated from bypass, and the operation was completed. Apart from temporary neurologic deficit, recovery was, again, uneventful and the patient was discharged 12 days later. Radiological follow-up 18 months later showed no evidence of pseudoaneurysm. Clinically he is well restituted, working full-time. 


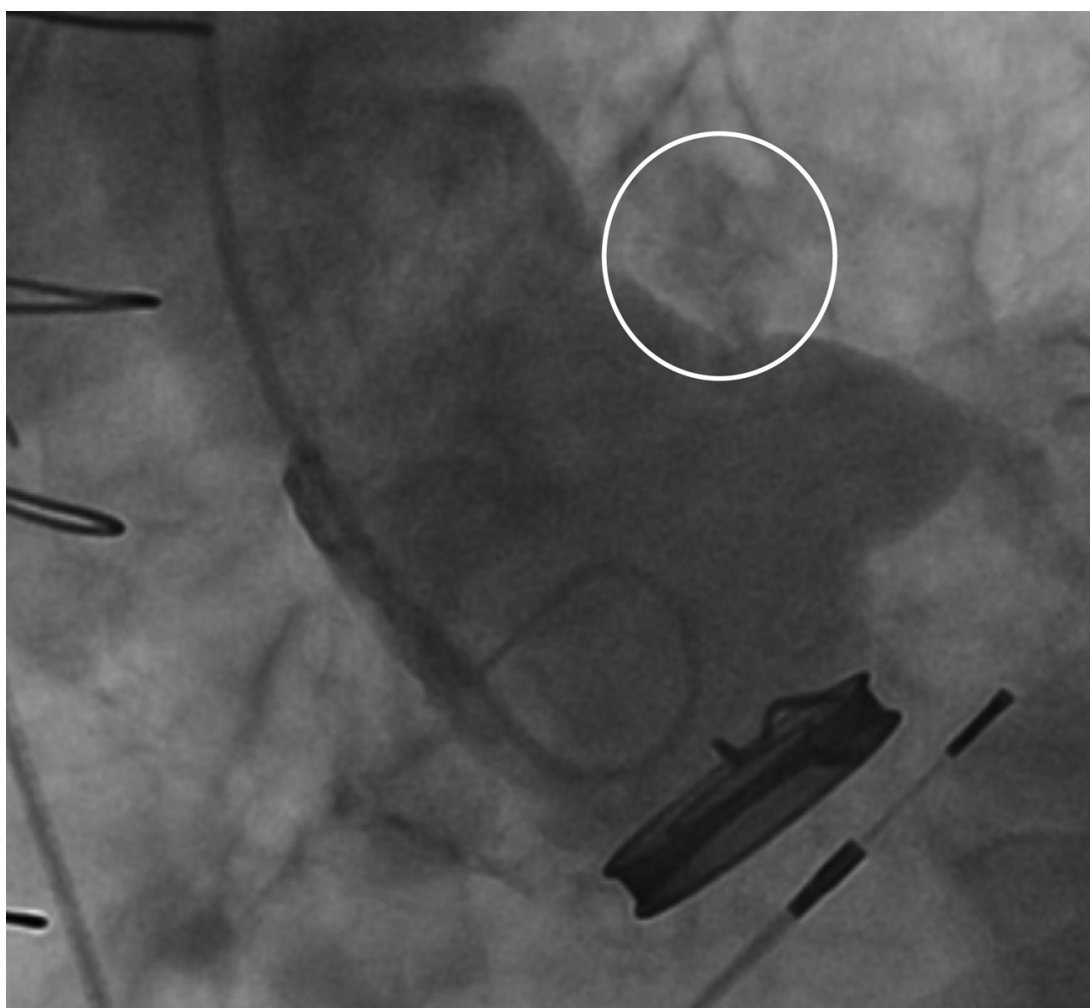

Figure 3. Angiography demonstrating contrast extravasation (inside white circle) from the left coronary artery button reimplantation.

\section{Discussion}

Aortic aneurysms and pseudoaneurysms may, rarely, erode the sternum or chest wall and present as "subcutaneous" bulging or swelling usually accompanied by pain [1]. In this particular patient, the sternum was not completely healed after a redo operation a year earlier (Fig. 2), allowing blood to seep painlessly between the sternal edges. Both CT and angiography were diagnostic of the underlying condition. Surgically, our institution's previously described strategy of preoperative circulatory arrest before resternotomy, without left ventricular decompression [2], proved efficient in avoiding foreseeable bleeding problems and in myocardial protection.

\section{Conflict of Interest}

The authors have no conflict of interest relevant to this publication.

\section{Comment on this Article or Ask a Question}

\section{REFERENCES}

1. El-Sayed Ahmad A, Papadopoulos N, Moritz A, Zierer A. Treatment of a giant ascending aortic pseudoaneurysm presenting as a presternal pulsatile protrusion almost perforating through the skin. Interact Cardiovasc Thorac Surg. 2013;16:903-905. 10.1093/icvts/ivt055

2. Möller F, Liska J, Lockowandt U, Samuelsson S, Franco-Cereceda A. Resternotomy using hypothermic circulatory arrest. J Card Surg.
2010;25:372-376. 10.1111/j.1540-8191.2010. 01014.x

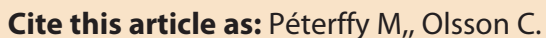
Second Redo for Composite Graft Pseudoaneurysm with Transcutaneous Rupture. Aorta 2014;2(5):207-210. DOI: http://dx. doi.org/10.12945/j.aorta.2014.14-026 


\section{EDITOR'S COMMENTS AND QUESTIONS}

\section{Editor's Comments}

The gross photograph of this pseudoaneurysm "necessitating" through the skin is dramatic. The authors' approach with pre-emptive cardiopulmonary bypass and circulatory arrest yielded an excellent clinical result.

Similar to the authors, we also have found that these coronary button pseudoaneurysms can be repaired through the pseudoaneurysm sac, without need to open the aorta itself.

\section{Editor's Questions}

(1) What is the root cause of these recurrent anastomotic aneurysms in this patient? Do you think it is infection? Was there any evidence of graft or suture degeneration? Do you happen to know if biological glue was used at the first operation?

While difficult to completely rule out, the clinical course and condition over a prolonged observation time has not been consistent with persisting composite graft infection. Apart from the extensive calcifications, there were no specific signs of tissue degeneration. However, as evident from the angiography image, the left coronary artery button was quite large. Whether this reflects the immediate result of the index surgical procedure or represents a gradual degeneration-a coronary button aneurysm-we do not know. Biological glue was not abandoned in our practice at the time of the index operation, but it has been used almost exclusively in acute dissections, to reapproximate and reinforce the dissected aortic tissue; it was not used in this patient. 